\title{
Circularly Polarized Luminescence and a Reflective Photoluminescent Chiral Nematic Liquid Crystal Display Based on an Aggregation-Induced Emission Luminogen
}

\author{
Dongyu Zhao, Hexiang He, Xinggui Gu, Lin Guo,* Kam Sing Wong, * Jacky W. Y. Lam, \\ and Ben Zhong Tang*
}

Chiral nematic liquid crystals $\left(\mathrm{N}^{*} \mathrm{LCs}\right)$ are a kind of smart material that can be obtained directly from cholesteric LCs or from nematic LCs by adding a chiral dopant. As a 1D photonic crystal, $^{[1]}$ the helical and periodic structure of a $\mathrm{N} * \mathrm{LC}$ leads to unique optical properties, including selective reflection, circular dichroism (CD), optical rotation, and so on. ${ }^{[2-5]}$ The most important property of $\mathrm{N}^{*} \mathrm{LCs}$ is the selective reflection of circularly polarized (CP) light. When $\mathrm{N} * \mathrm{LCs}$ are sandwiched between two substrates with planar anchoring surfaces, CP light with the same handedness as the helix will be selectively reflected whereas light with opposite handedness will be transmitted. The maximum or center wavelength $\lambda$ of the selective reflection is generally defined as

$\lambda=n P$

where $P$ is the helical pitch length of the structure and $n$ is the average refractive index of the LC material. The stop band is another representation of the selective reflection band of a $\mathrm{N}^{*} \mathrm{LC}$, and the band width $(\Delta \lambda)$ is equal to $\Delta n P$, where $\Delta n$ is the optical anisotropy. If the reflective wavelength $\lambda$ is in the visible light range, colored light will be selectively reflected. The applications of the unique optical properties of the $\mathrm{N}^{*} \mathrm{LC}$ helical structure have been explored in diverse techniques, ${ }^{[6]}$ such as polarizerfree reflective displays, ${ }^{[7,8]}$ polarizers, ${ }^{\left[{ }^{[9]}\right.}$ reflectors, ${ }^{[10]}$ and optical sensors. ${ }^{[11]}$ Conventional reflective LCDs fabricated with $\mathrm{N}^{*} \mathrm{LCs}$ usually employ an external light source to provide outdoor images with vivid colors. ${ }^{[6,7,12,13]}$ However, these reflective LCDs are not

\section{Dr. D. Y. Zhao, Prof. L. Guo \\ Key Laboratory of Bio-Inspired Smart Interfacial Science and Technology of the Ministry of Education School of Chemistry and Environment Beihang University \\ Beijing 100191, P. R. China \\ E-mail: guolin@buaa.edu.cn; phkswong@ust.hk}

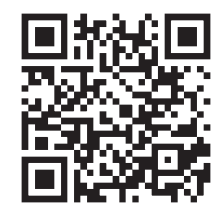

Dr. D. Y. Zhao, Dr. X. G. Gu, Dr. J. W. Y. Lam, Prof. B. Z. Tang

Department of Chemistry

Institute for Advanced Study

Institute of Molecular Functional Materials

and Division of Biomedical Engineering

The Hong Kong University of Science \& Technology (HKUST)

Clear Water Bay, Kowloon, Hong Kong, P. R. China

E-mail: tangbenz@ust.hk

Dr. H. X. He, Prof. K. S. Wong

Department of Physics

the Hong Kong University of Science \& Technology (HKUST)

Clear Water Bay, Kowloon, Hong Kong, P. R. China

DOI: 10.1002/adom.201500646 functional when there is no ambient light as LCs are not emitting structures. Therefore, in dark environments, a light source is required for $\mathrm{N} * \mathrm{LC}$ displays. Moreover, a color filter and a set of polarizers are also necessary, which are hugely energy consuming because of their limited transmittance of the incident light. ${ }^{[14,15]}$

In order to overcome these shortcomings, developing photoluminescent $\mathrm{N} * \mathrm{LCs}$ that combine excellent luminescent properties with LC supramolecular organization is an attractive approach. ${ }^{[16-18]}$ Doping fluorescent dyes into $N *$ LC hosts is an effective way to obtain circularly polarized luminescence (CPL), which has potential applications in organic light-emitting diodes (OLEDs), lasing materials, and as optical amplifiers. ${ }^{[19-24]}$ The emission spectra can thus be modulated by tuning the photonic stop band. Inside the stop band, propagation of CPL with the same handedness as the helix is suppressed, leading to a higher degree of CPL opposite to the helix handedness in this region. Up until now, organic luminescent dyes have been mostly used as the fluorescent dopant to fulfill the demands of CPL studies because of their tailored synthetic feasibility, low cost, and high processability. ${ }^{[22,25-31]}$ Unfortunately, a serious problem associated with most luminophores is the intrinsic self-quenching. This is induced by the high concentration of fluorescent additives due to $\pi-\pi$ stacking interactions in the aggregated state, known as aggregation-caused quenching (ACQ), ${ }^{[32-34]}$ which has significantly limited the use of luminogens in optoelectronic devices. As an alternative to conventional organic dyes, some inorganic quantum dots (QDs) with relatively narrow emission bands and high brightness have been introduced to obtain wavelengthtunable CPL, thus avoiding emission quenching. ${ }^{[35-41]}$ Despite these advantages, the main problem of embedding QDs into the LC medium is that it is hard to attain a high concentration of QDs while retaining the optical properties. Another problem is that QDs tend to aggregate inside the LCs. Therefore, only a few studies have been reported on QD-N*LC-based CPL so far.

Recently, a new class of non-planar organic luminogens with aggregate-induced emission (AIE) properties has attracted tremendous attention. ${ }^{[42-47]}$ As a fantastic photophysical phenomenon, AIE is diametrically opposed to the ACQ effect. Usually containing a structure with molecular rotors, AIE molecules are weakly fluorescent or non-fluorescent in the solution state due to active intramolecular rotation that consumes energy from the excited state, but become brightly fluorescent induced by aggregation or high viscosity. It has been found that AIE molecules emit more efficiently in a more viscous solvent, at lower temperature, and under higher pressure. Through systematic studies, restriction of intramolecular rotation (RIR) and intramolecular vibrations (RIV) have been identified as the two 


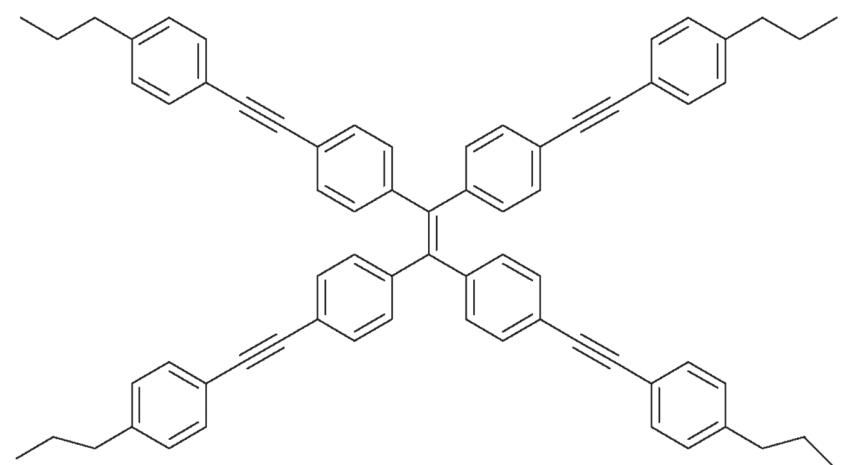

Scheme 1. The chemical structure of TPE-PPE.

main causes for the AIE effect. Suppression of the molecular motions activates the radiative decay channel, making AIE molecules highly emissive in the aggregated state. The discovery of the intriguing AIE phenomenon furnishes the possibility to conquer the quenching problem and shows real potential applications in organic light-emitting materials, ${ }^{[44]}$ OLEDs, ${ }^{[45]}$ bioprobes, ${ }^{[46]}$ and fluorescent nanocapsules, ${ }^{[47]}$ etc.

Recently, we synthesized an AIE-LC compound, namely tetraphenylethylene-propylphenylethyne (TPE-PPE) ${ }^{[48]}$ as shown in Scheme 1. In this work, we doped TPE-PPE into chiral nematic LCs and studied the CPL performance of the LC composites. It was revealed that introducing TPE-PPE into the $\mathrm{N} * \mathrm{LC}$ medium had no influence on the CPL degree of the LC composites but played a key role in determining the emission intensity due to the AIE effect in the system. Moreover, a reflective-luminescent $\mathrm{N} * \mathrm{LC}$ display device based on the selective reflection and CPL behavior was constructed. This device could work in different lighting conditions, from direct sunlight to total darkness. This reflective-luminescent $\mathrm{N} * \mathrm{LC}$ display simplified the device design and reduced the energy consumption by saving not only on the backlight source but also the color filter, providing a promising prospect for future energy-saving displays that can be used under different lighting conditions.

First, the influence of TPE-PPE on the selective reflection of the $\mathrm{N} * \mathrm{LCs}$ was investigated. A series of $\mathrm{N} * \mathrm{LC}$ samples in the visible light range were prepared by mixing a nematic LC and the right-handed chiral dopant CB15 in different concentrations. Photoluminescent (PL)- $\mathrm{N}^{*} \mathrm{LC}$ samples were then obtained by doping 0.5 wt $\%$ TPE-PPE into the $\mathrm{N} *$-LC hosts. The solid and dashed curves in Figure 1a indicate the reflection spectra of the planar aligned $\mathrm{N}^{*} \mathrm{LC}$ and PL-N*LC cells, respectively. The similar reflection wavelength of the $\mathrm{N} * \mathrm{LC}$ and PL$\mathrm{N} *$-LC cells indicates that doping with $0.5 \mathrm{wt} \%$ of TPE-PPE did not change the stop band significantly. Figure $1 \mathrm{~b}$ shows the photos of $\mathrm{N} * \mathrm{LC}$ and PL-N*LC cells, which exhibit the similar colors under daylight. In addition, the TPE-PPE concentration was also varied to study if it changed the selective reflection. As reported in Figure S1 (Supporting Information), there was no change in the reflection spectra, suggesting that the concentration of TPE-PPE had no influence on the reflection. After that, the $\mathrm{N} * \mathrm{LC}$ and PL-N*LC cells were investigated with CD spectroscopy, which measures the differential absorption of lefthanded (L) CP and right-handed (R) CP light. In this case, the difference in absorption is due to the difference in reflection of the bandgap region. As shown in Figure 1c, the N*LC cells showed a distinct Cotton effect with a negative signal (solid curves) at their stop band regions, demonstrating that rightcircularly polarized (RCP) light was selectively reflected by the right-handed $\mathrm{N} * \mathrm{LC}$ structure. For the PL-N*LC cells (dashed curves), a similar line shape was observed and the peaks appeared in the same regions as those of the $\mathrm{N} * \mathrm{LC}$ samples, matching their stop bands. The CD spectra results indicate that the $\mathrm{N}^{*}$-LC and PL-N*-LC cells have the same helical sense of chirality and the observed colors in both cases arose from the selective reflection of the circularly polarized light. Moreover, to further demonstrate the handedness of the $\mathrm{N}^{*}$-LC and PL$\mathrm{N} *$-LC samples, images of the $\mathrm{N} * \mathrm{LC}$ and PL-N*LC cells under a polarizer are provided in Figure S2 (Supporting Information). The polarizer used here had two windows for either left- or right-handed polarized light, which was prepared by stacking multi-layer polyvinyl alcohol (PVA) films on a triacetyl cellulose (TAC) substrate, followed by drying and a uniaxial tension process. It can be seen that both $\mathrm{N} * \mathrm{LC}-3$ and PL-N*LC-3 cells exhibited a transmitted image through the left-handed polarizer window (Figure S2a) and a reflected color image from the righthanded polarizer window (Figure S2b), indicating clearly that the $\mathrm{N} * \mathrm{LC}-3$ and PL-N*LC-3 cells have the same right-handed
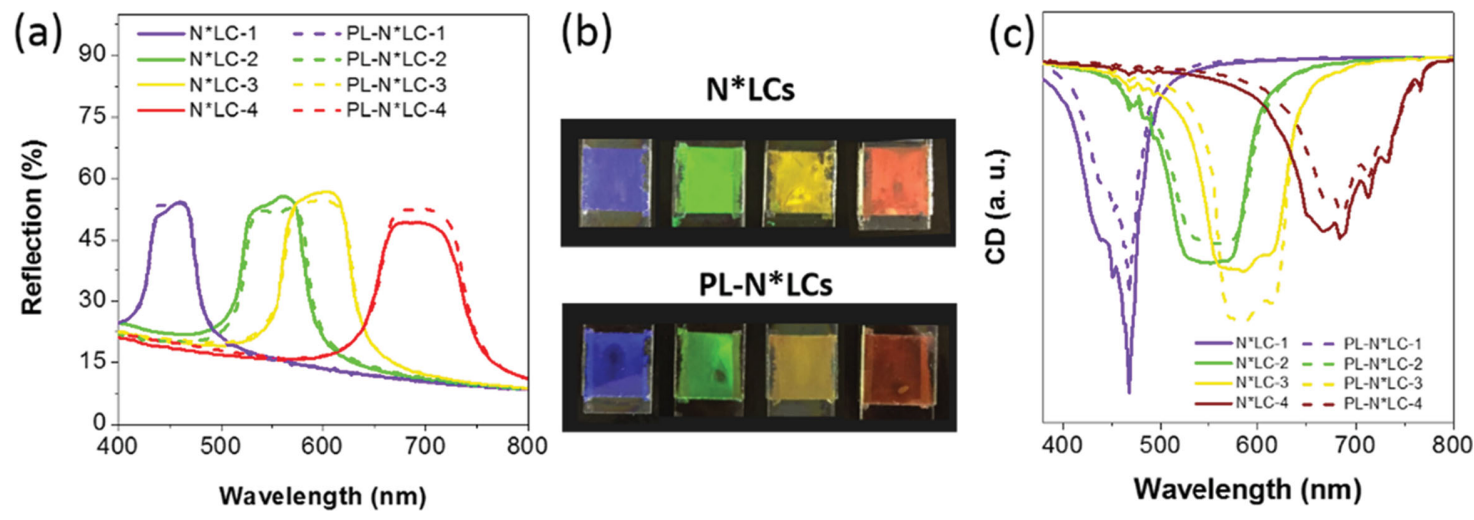

Figure 1. a) Reflection spectra of right-handed circularly polarized light of the $\mathrm{N} * \mathrm{LC}$ (solid lines) and PL-N*LC (dashed lines) cells. In the PL-N*LC cells, the concentration of TPE-PPE in the $\mathrm{N} * \mathrm{LC}$ host was $0.5 \mathrm{wt} \%$. b) Photos of $\mathrm{N} * \mathrm{LC}$ cells and PL-N*LCs cells in a planar alignment under daylight irradiation. c) CD spectra of the $\mathrm{N} * \mathrm{LC}$ (solid lines) and PL-N*LC (dashed lines) cells. 

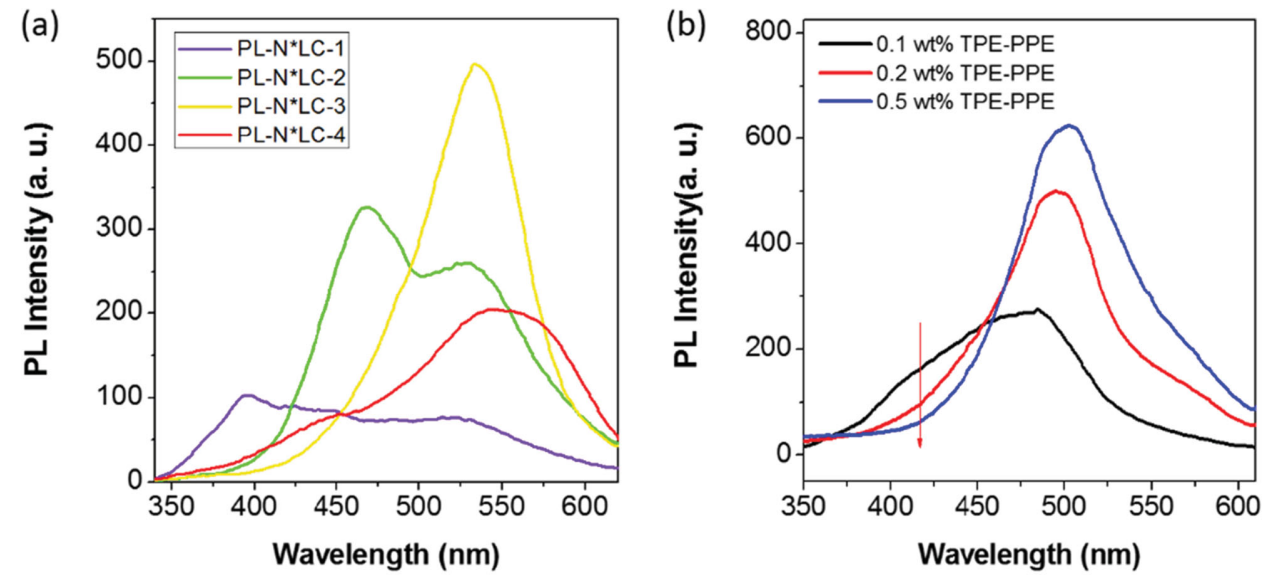

Figure 2. a) Non-polarized PL spectra of the PL- $N * L C$ cells at different reflection wavelengths. The concentration of TPE-PPE was 0.5 wt $\%$. b) PL spectra of the PL-N*LC cells composed of $\mathrm{N} * \mathrm{LC}-3$ and TPE-PPE with concentrations of $0.1 \mathrm{wt} \%, 0.2 \mathrm{wt} \%$, and $0.5 \mathrm{wt} \%$.

helix structure. This result is in accordance with the above CD spectral characteristics.

After proving that the TPE-PPE film had no influence on the selective reflection, we wondered whether the selective reflection influences the luminescence of the light-emitting LC mixtures. Firstly, the non-polarized PL spectra were studied. Figure 2a shows the PL spectra of the PL-N*LC cells. Figure S3 in the Supporting Information shows the emission spectrum of TPE-PPE, whose maximum emission wavelength was at around $525 \mathrm{~nm}$ (Figure S3a), and the PL spectra of the $\mathrm{N} * \mathrm{LC}$ cells (Figure S3b). It can be seen that all four $\mathrm{N}^{*} \mathrm{LC}$ samples had a broad PL spectrum from $350 \mathrm{~nm}$ to $550 \mathrm{~nm}$. After incorporating the PL spectra of the $\mathrm{N}^{*} \mathrm{LCs}$ with those of the TPE-PPE film, the PL spectra of the PL-N*LCs were found to be the superposition of that of the $\mathrm{N} * \mathrm{LCs}$ and TPE-PPE, as shown in Figure S4a-d (Supporting Information). Moreover, the emission intensity of the PL-N*LC- 2 and PL-N*LC- 3 cells shown in Figure 2a were higher than that of the PL- $\mathrm{N}^{*} \mathrm{LC}-1$ and PL-N*LC-4 cells, resulting from the fact that there was more overlap between the emission wavelength of TPE-PPE and the photonic stop band (reflection band) of the $\mathrm{N} * \mathrm{LC}-2$ and $\mathrm{N} * \mathrm{LC}-3$ cells. Within the stop band of the $\mathrm{N}^{*} \mathrm{LCs}$, the emission of TPEPPE will be reflected, so that the detected PL intensity will be stronger. In other words, for the PL-N*LCs, the emission wavelength depends on both the emission wavelength of the TPEPPE and that of the $\mathrm{N} * \mathrm{LCs}$ whereas the emission intensity depends only on the relative positions of the photonic stop band of the $\mathrm{N} * \mathrm{LCs}$ and that of the emission wavelength of TPE-PPE.

Figure $2 \mathrm{~b}$ shows the PL spectra of the LC samples composed of $\mathrm{N} * \mathrm{LC}-3$ and different concentrations of TPE-PPE. With increasing concentration of luminescent TPE-PPE, the emission intensity also increased. This phenomenon results from the AIE effect in our system: instead of being quenched the TPE-PPE emits intensely when its molecules tend to aggregate with increasing concentration in the LC host. It should be noted that at a wavelength of around $420 \mathrm{~nm}$ the PL intensity decreased with increasing TPE-PPE concentration, resulting from the energy transfer between the $\mathrm{N} * \mathrm{LC}-3$ and TPE-PPE in the system, as shown in Figure S5a (Supporting Information). In addition, in order to undertake extensive research on the AIE effect in our system, we utilized a conventional fluorophore, fluorescein, for comparison. It can be seen from Figure S5b that the emission intensity of the LC cell composed of $\mathrm{N} * \mathrm{LC}-3$ and fluorescein decreased intensively with increasing fluorescein concentration, implying that the emission was quenched caused by the aggregation of fluorescein during this process. This further indicates that AIE plays a prominent role in the emission of $\mathrm{N} * \mathrm{LC} / \mathrm{TPE}-\mathrm{PPE}$.

After these investigations, CPL measurements were performed; a schematic view of the setup is shown in Figure S6 and the CPL results of the PL-N*LC cells are given in Figure S7 in the Supporting Information. The degree of CPL is defined by the circular dissymmetry factor

$$
\mathrm{g}_{\mathrm{e}}=2\left(I_{\mathrm{L}}-I_{\mathrm{R}}\right) /\left(I_{\mathrm{L}}+I_{\mathrm{R}}\right)
$$

where $I_{\mathrm{L}}$ and $I_{\mathrm{R}}$ refer to the intensity of the left and right CPL, respectively. Figure S7a (Supporting Information) shows the $g_{\mathrm{e}}$, alternating current $(\mathrm{AC})\left(I_{\mathrm{L}}-I_{\mathrm{R}}\right)$, and direct current (DC) $\left(\left(I_{\mathrm{L}}+I_{\mathrm{R}}\right) / 2\right)$ curves of PL-N*LC-1, whose center reflection wavelength was at around $450 \mathrm{~nm}$. It can be found from the $\mathrm{g}_{\mathrm{e}}$ curve that the PL-N*LC-1 sample emitted L-CPL within the stop band, owing to the 1D photonic crystal characteristics. In the right-handed $\mathrm{N} * \mathrm{LC}$ structure, propagation of the R-CPL was suppressed within the stop band and the L-CPL was transmitted through the cell. The AC result also confirmed this theory. There is a bulge in the AC curve in this region because $I_{\mathrm{L}}$ represents the PL spectrum without photonic effects whereas $I_{R}$ is suppressed because of the stop band, leading to an increase of the AC value. Figure S7b-d (Supporting Information) also showed similar results. Moreover, it can be observed that the $g_{e}$ curve displayed a sharp and precisely signal reversal at the edge of the stop band, due to the enhanced density of state (DOS) present at the stop band edge of the photonic crystal.[25,49] Figure 3 shows the CPL results of $\mathrm{N} * \mathrm{LC}-3$ doped with 0.1 wt\%, $0.2 \mathrm{wt} \%$, and $0.5 \mathrm{wt} \%$ TPE-PPE. It was found that the $g_{e}$ values. were quite similar, indicating that the degree of CPL is not related to the TPE-PPE concentration. On the other hand, the DC value at a wavelength of around $530 \mathrm{~nm}$ increased rapidly with increasing concentration of TPE-PPE, due to the AIE effect of TPE-PPE in the LC hosts, similar to the results of the 

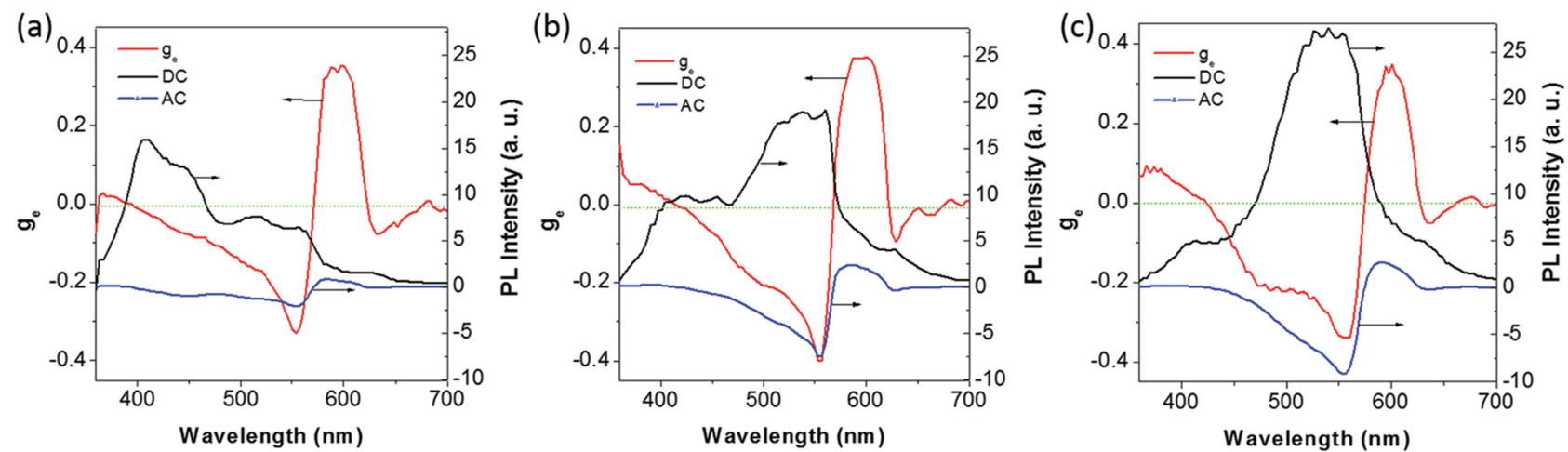

Figure 3. Emission dissymmetry factor $\left(g_{e}\right), A C$, and $D C$ curves of the PL-N*LC cells fabricated with $N * L C-3$ and TPE-PPE with concentrations of: a) $0.1 \mathrm{wt} \%$, b) $0.2 \mathrm{wt} \%, \mathrm{c}) 0.5 \mathrm{wt} \%$. AC $=I_{\mathrm{L}}-I_{R}, \mathrm{DC}=\left(I_{\mathrm{L}}+I_{R}\right) / 2, g_{\mathrm{e}}=\mathrm{AC} / \mathrm{DC}$. Ratio of $\mathrm{N} * \mathrm{LC}-3$ : SCL1717/CB15 $=0.64 / 0.36$. The wavelength of the excitation light was $325 \mathrm{~nm}$.

non-polarized PL experiments in Figure 2b. These results indicate that although the TPE-PPE concentration had no effect on the degree of CPL, it did, however, determine the emission intensity of the $\mathrm{N} * \mathrm{LC}$ mixture, because of the AIE effect in the $\mathrm{N} * \mathrm{LC}$ medium.

Based on the above results, we chose the PL-N*LC-3 structure, whose selective light reflection band was between 570 and $620 \mathrm{~nm}$ and contained $0.5 \mathrm{wt} \%$ TPE-PPE, to study the CPL under application of a DC electric field to the cell. As recorded in Figure 4a, when the electric field was off, the orientation of the LC mixture was parallel to the substrate surface and the LC cell emitted L- and R-CPL simultaneously. When the applied electric field reached $60 \mathrm{~V}$, the AC curve changed to a straight line and the value of $g_{e}$ became almost zero, as shown in Figure $4 \mathrm{~b}$, meaning that the $I_{R}$ value is close to the $I_{\mathrm{L}}$ value, because of the homeotropic alignment of the LC molecules under the high voltage of the electric field. Therefore, the CPL disappeared and the LC cell emitted non-polarized light. Moreover, when the electric field was switched on, the PL intensity, which can be defined by the value of the DC curve, dropped by about two thirds compared to that in the electric-field off state. When the DC voltage was turned back off, the polarization of the fluorescence was recovered within several minutes.

Based on the selective reflection and CPL properties of the PL-N*LCs, an electric-controlled LC cell was fabricated. Figure 5 shows a schematic representation of the mechanism, the microscopy images of the textures observed by polarized optical microscopy (POM), and photos of the PL-N*LC-3 cell with and without an electric field. Normal sunlight and UV light were used as the light sources for the day- and nighttime scenarios, respectively. A right-handed circular polarizer was used to ensure that the R-CPL was transmitted. As shown in Figure 5a, the $\mathrm{N} *$-LC molecules were aligned parallel to the substrate surface in the initial state. Under sunlight irradiation, the color due to the Bragg reflection of the right-handed helical structure could be seen through the cell and the polarizer; whereas under UV-light irradiation, the R-CPL was transmitted through the right-handed circular polarizer. Figure $5 \mathrm{c}$ and $5 \mathrm{e}$ show the POM images of the cell under sunlight and UV light irradiation, respectively, in the initial state (electric field off). The inset of Figure $5 c$ shows a photo of the cell where a yellow color can be seen that is due to the selective reflection of $\mathrm{N} * \mathrm{LC}-3$. A photo under UV light irradiation is given in the inset of Figure $5 \mathrm{e}$. When applying a direct current (DC) electric field to the cell, the helical twist was distorted so strongly that the Bragg reflection was completely eliminated and the alignment of the LC molecules changed to a vertical one, as illustrated in Figure 5b. As a result, no reflection light could be observed under sunlight irradiation, as shown in Figure 5d. Whereas under UV light irradiation, the CPL switched to a non-polarized state under the electric field and a
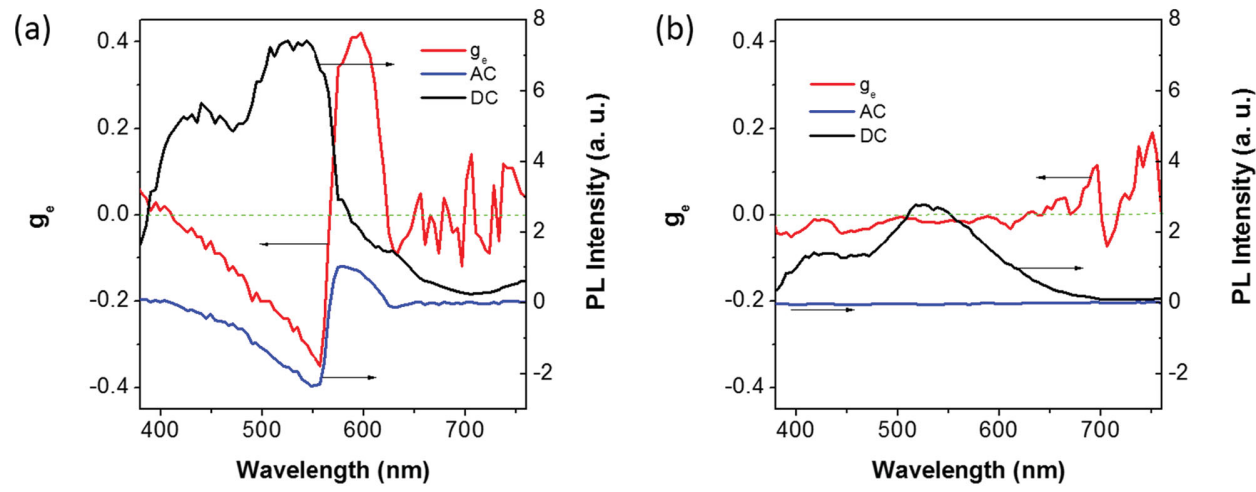

Figure 4. Emission dissymmetry factors $\left(g_{e}\right)$, DC, and AC spectra of the PL-N*LC-3 cell with the electric field (60 V DC voltage) in the: a) "off" and b) "on" state. The wavelength of the excitation light was $325 \mathrm{~nm}$. 
(a)

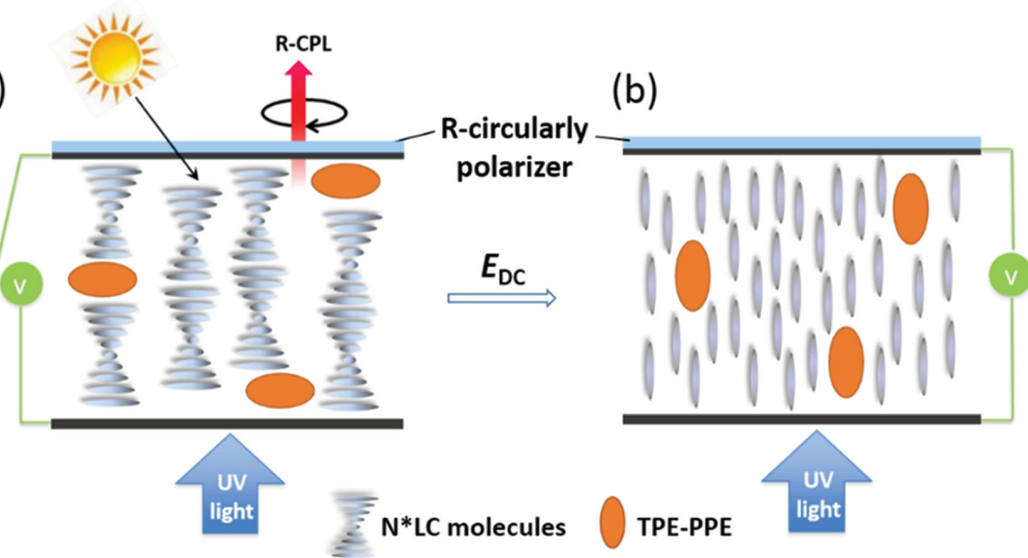

(c)

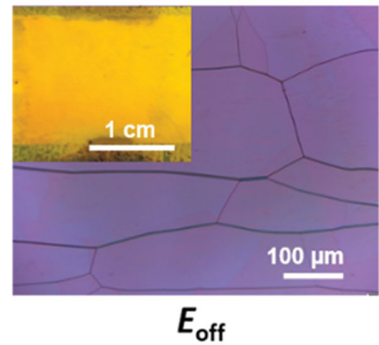

(e)

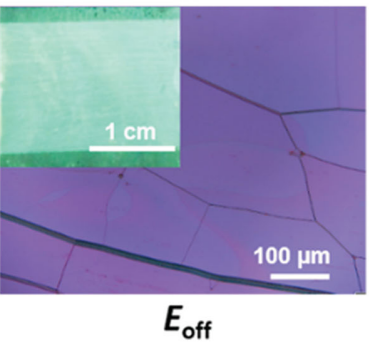

(d)

Under day light

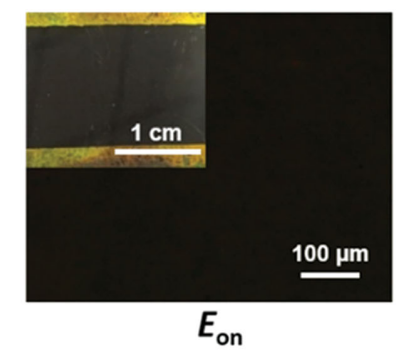

(f)

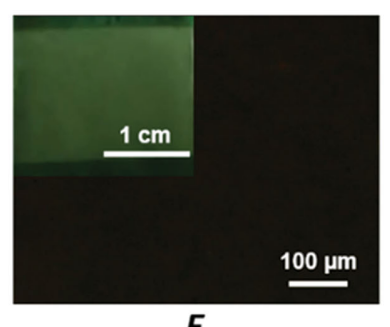

Under UV light

$E_{\text {on }}$

Owing to the selective reflection and emissive properties of PL-N*LCs, a reflective-luminescent LC display device was constructed on a patterned ITO glass substrate. To test the performance of the device, sunlight and a UV lamp were used as the light sources during the day and night time, respectively. A righthanded circular polarizer was used to ensure that R-CP light was transmitted through the cell. When a DC electric field $(60 \mathrm{~V})$ was applied to the LC cell, the molecules in the region with ITO changed from a planar orientation to a homeotropic alignment. As a result, the region without ITO exhibited the reflected color under sunlight whereas the same region emitted R-CP light under UV irradiation through the right-handed circular polarizer. Figure $\mathbf{6}$ shows a photograph of the reflective-luminescent LC display device as perceived by the eye, showing the display switching from a daytime to a night time environment. It is clear that this device works in both daytime under sunlight and at night under UV light irradiation.

In summary, we have prepared PL-N*LCs by mixing a series of $\mathrm{N}^{*} \mathrm{LCs}$ and an AIE luminogen. The selective reflection and CPL properties as well as their interaction were studied. The AIE effect is fully implemented in our system, which will facilitate the application of AIE luminogens in LC displays. Under application of a DC electric field, the CPL disappeared and the PL intensity changed due to the electric-field-induced homeotropic orientation of the LC molecules. Thanks to this switching mechanism, a reflective-photoluminescent $\mathrm{N} * \mathrm{LC}$ display device that works under both sunlight and UV light irradiation was constructed. This work therefore provides promising prospects for future energy-saving displays that can be used under different lighting conditions.

Figure 5. Schematic representation of the molecular arrangements, POM images, and photographs of the PL-N*LC cell under sunlight and UV light irradiation using a light-emitting $\mathrm{N} * \mathrm{LC}$ mixture ( $\mathrm{N} * \mathrm{LC}-3+0.5$ wt\% TPE-PPE). a,b) Illustration of molecular arrangements of the PL-N*LC cell with the electric field "off" and "on", respectively. c,d) POM images of the PL$\mathrm{N} * \mathrm{LC}$ cell under sunlight irradiation. Inset: Photos of the cell with the electric field "off" and "on", respectively. e,f) POM images of the PL-N*LC cell under UV light irradiation at $365 \mathrm{~nm}$. Inset: Photos of the cell with the electric field "off" and "on", respectively. The wavelength of the excitation light was $365 \mathrm{~nm}$.

non-polarized emission with low intensity could be observed, as shown in Figure $5 f$. A photo of the cell under UV light and with applied electric field is shown in the inset of Figure $5 \mathrm{f}$.

\section{Supporting Information}

Supporting Information is available from the Wiley Online Library or from the author.

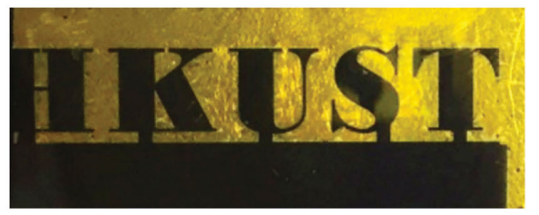

Under Sunlight

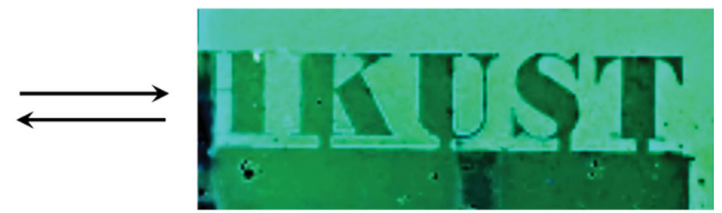

Under UV light

Figure 6. Photographs of the reflective-luminescent display device with an applied DC electric field (60 V) under sunlight and under UV-light irradiation using the light-emitting $\mathrm{N} * \mathrm{LC}$ mixture. The LC mixture $=\mathrm{N} * \mathrm{LC}-3+0.5$ wt\% TPE-PPE. The wavelength of the excitation light was $365 \mathrm{~nm}$. 


\section{Acknowledgements}

This work was partially supported by the National Natural Science Foundation of China (51203005), the National Basic Research Program of China (973 Program, 2013CB834701), the Research Grants Council of Hong Kong (16305015, 16301614 and N_HKUST604/14), the University Grants Committee of Hong Kong (AoE/P-03/08) and the New Teacher Fund for Doctor Station, the Ministry of Education of China (20121102120045). B. Z. T. thanks the support of the Guangdong Innovative Research Team Program (201101C0105067115). K. S. W. thanks the support of the Research Grants Council of Hong Kong (project AoE/P-02/12 and CUHK1/CRF/12G).

Received: November 1, 2015 Published online: January 8, 2016

[1] J. W. Goodby, P. J. Collings, T. Kato, C. Tschierske, H. Gleeson, P. Raynes, Handbook of Liquid Crystals, 2nd edition, Wiley-VCH, Weinheim, Germany 2014.

[2] J. Fan, Y. Li, H. K. Bisoyi, R. S. Zola, D. K. Yang, T. J. Bunning, D. A. Weitz, Q. Li, Angew. Chem. Int. Ed. 2015, 54, 2160.

[3] L. Wang, H. Dong, Y. Li, C. Xue, L. D. Sun, C. H. Yan, Q. Li, J. Am. Chem. Soc. 2014, 136, 4480.

[4] H. K. Bisoyi, Q. Li, Acc. Chem. Res. 2014, 47, 3184.

[5] H. Wang, L. Wang, H. Xie, C. Li, S. Guo, M. Wang, C. Zou, D. Yang, H. Yang, RSC Adv. 2015, 5, 33489.

[6] M. Mitov, Adv. Mater. 2012, 24, 6260.

[7] Q. Li, Y. Li, J. Ma, D. K. Yang, T. J. White, T. J. Bunning, Adv. Mater. 2011, 23, 5069 .

[8] J. K. Kim, S. H. Joo, J. K. Song, Appl Opt. 2013, 52, 8280.

[9] Y. Huang, Y. Zhou, S.-T. Wu, Opt. Express 2007, 15, 6414.

[10] N. Y. Ha, Y. Ohtsuka, S. M. Jeong, S. Nishimura, G. Suzaki, Y. Takanishi, K. Ishikawa, H. Takezoe, Nat. Mater. 2008, 7, 43.

[11] D. J. Mulder, A. P. H. J. Schenning, C. W. M. Bastiaansen, J. Mater. Chem. C 2014, 2, 6695

[12] B.-Y. Lee, J.-H. Lee, Curr. Appl. Phys. 2011, 11, 1389.

[13] K.-H. Kim, B.-H. Yu, S.-W. Choi, S.-W. Oh, T.-H. Yoon, Opt. Express 2012, 20, 24376.

[14] K. Binnemans, C. Görller-Walrand, Chem. Rev. 2002, 102, 2303.

[15] D. Bloor, R. W. Cahn, The Encyclopedia of Advanced Materials, Pergamon, New York, NJ 1994.

[16] B. A. San Jose, J. Yan, K. Akagi, Angew. Chem. Int. Ed. 2014, 53, 10641

[17] W.-Z. Zhao, Y.-H. Cong, B.-Y. Zhang, T.-H. Zhang, Liq. Cryst. 2014, $41,1762$.

[18] K. Watanabe, I. Osaka, S. Yorozuya, K. Akagi, Chem. Mater. 2012, 24, 1011.

[19] S. Chen, D. Katsis, A. Schmid, J. Mastrangelo, T. Tsutsui, T. Blanton, Nature 1999, 397, 506.

[20] Q. Ye, D. Zhu, H. Zhang, X. Lu, Q. Lu, J. Mater. Chem. C 2015, 3, 6997.
[21] E. M. Sanchez-Carnerero, F. Moreno, B. L. Maroto, A. R. Agarrabeitia, M. J. Ortiz, B. G. Vo, G. Muller, S. de la Moya, J. Am. Chem. Soc. 2014, 136, 3346.

[22] T. Hamamoto, M. Funahashi, J. Mater. Chem. C 2015, 3, 6891.

[23] H. Li, J. Cheng, Y. Zhao, J. W. Y. Lam, K. S. Wong, H. Wu, B. S. Li, B. Z. Tang, Mater. Horizons 2014, 1, 518.

[24] J. Park, T. Yu, T. Inagaki, K. Akagi, Macromolecules 2015, 48, 1930.

[25] A. Y. Bobrovsky, N. I. Boiko, V. Shibaev, J. H. Wendorff, Adv. Mater. 2003, 15, 282.

[26] J. r. Schmidtke, G. Jünnemann, S. Keuker-Baumann, H.-S. Kitzerow, Appl. Phys. Lett. 2012, 101, 051117.

[27] S. Furumi, Y. Sakka, Adv. Mater. 2006, 18, 775.

[28] H. Lu, L. Qiu, G. Zhang, A. Ding, W. Xu, G. Zhang, X. Wang, L. Kong, Y. Tian, J. Yang, J. Mater. Chem. C 2014, 2, 1386.

[29] Z. Wang, C. Yang, W. Li, L. Chen, X. Wang, Z. Cai, Appl. Phys. B 2013, 115, 483.

[30] M. Voigt, M. Chambers, M. Grell, Liq. Cryst. 2002, 29, 653.

[31] M. Voigt, M. Chambers, M. Grell, Chem. Phys. Lett. 2001, 347, 173.

[32] Y. Hong, J. W. Lam, B. Z. Tang, Chem. Commun. 2009, 4332.

[33] Y. Hong, J. W. Lam, B. Z. Tang, Chem. Soc. Rev. 2011, 40, 5361.

[34] I. Ilchishin, L. Lysetskiy, T. Mykytiuk, M. Serbina, Opt.. Mater. Express 2011, 1, 1484

[35] A. Bobrovsky, K. Mochalov, V. Oleinikov, A. Sukhanova, A. Prudnikau, M. Artemyev, V. Shibaev, I. Nabiev, Adv. Mater. 2012, 24, 6216.

[36] A. Bobrovsky, P. Samokhvalov, V. Shibaev, Adv. Opt. Mater. 2014, 2, 1167.

[37] G. Petriashvili, G. Chilaya, M. A. Matranga, M. P. D. Santo, G. Cozza, R. Barberi, J. d. Barrio, L. S. Chinelatto, L. Oriol, M. Piñol, Opt. Mater. 2009, 31, 1693.

[38] A. Bobrovsky, K. Mochalov, V. Oleinikov, V. Shibaev, Liq. Cryst. 2011, 38, 737.

[39] L.-J. Chen, C.-R. Lee, C.-L. Chu, RSC Adv. 2014, 4, 52804.

[40] L.-J. Chen, J.-D. Lin, C.-R. Lee, J. Mater. Chem. C 2014, 2, 4388.

[41] I. C. Khoo, K. E. Mochalov, A. Y. Bobrovsky, V. A. Oleinikov, A. V. Sukhanova, A. E. Efimov, V. Shibaev, I. Nabiev, Proc. SPIE 2012, 8475,847514

[42] J. Luo, Z. Xie, J. W. Y. Lam, L. Cheng, B. Z. Tang, H. Chen, C. Qiu, H. S. Kwok, X. Zhan, Y. Liu, D. Zhu, Chem. Commun. 2001, 381, 1740.

[43] J. Mei, N. L. C. Leung, R. T. K. Kwok, J. W. Y. Lam, B. Z. Tang, Chem. Rev. 2015, 115, 11718.

[44] Z. Xie, C. Chen, S. Xu, J. Li, Y. Zhang, S. Liu, J. Xu, Z. Chi, Angew. Chem. Int. Ed. 2015, 54, 7181.

[45] G. Chen, W. Li, T. Zhou, Q. Peng, D. Zhai, H. Li, W. Z. Yuan, Y. Zhang, B. Z. Tang, Adv. Mater. 2015, 27, 4496.

[46] E. Zhao, Y. Chen, S. Chen, H. Deng, C. Gui, C. W. Leung, Y. Hong, J. W. Lam, B. Z. Tang, Adv. Mater. 2015, 27, 4931

[47] Y. Okazawa, K. Kondo, M. Akita, M. Yoshizawa, J. Am. Chem. Soc. 2015, 137, 98

[48] D. Zhao, F. Fan, J. Cheng, Y. Zhang, K. S. Wong, V. G. Chigrinov, H. S. Kwok, L. Guo, B. Z. Tang, Adv. Opt. Mater. 2015, 3, 199.

[49] M. Chambers, M. Voigt, M. Grell, Chem. Phys. Lett. 2002, 355, 214. 